\title{
Mechanical Response of Hollow Metallic Nanolattices: Combining Structural and Material Size Effects
}

\author{
L. C. Montemayor \\ Division of Engineering and Applied Science, \\ California Institute of Technology, \\ Pasadena, CA 91125 \\ J. R. Greer \\ Division of Engineering and Applied Science, \\ California Institute of Technology, \\ Pasadena, CA 91125 \\ e-mail: jrgreer@caltech.edu
}

\begin{abstract}
Ordered cellular solids have higher compressive yield strength and stiffness compared to stochastic foams. The mechanical properties of cellular solids depend on their relative density and follow structural scaling laws. These scaling laws assume the mechanical properties of the constituent materials, like modulus and yield strength, to be constant and dictate that equivalent-density cellular solids made from the same material should have identical mechanical properties. We present the fabrication and mechanical properties of three-dimensional hollow gold nanolattices whose compressive responses demonstrate that strength and stiffness vary as a function of geometry and tube wall thickness. All nanolattices had octahedron geometry, a constant relative density, $\rho \sim 5 \%$, a unit cell size of 5-20 $\mu \mathrm{m}$, and a constant grain size in the Au film of 25-50 nm. Structural effects were explored by increasing the unit cell angle from 30 deg to 60 deg while keeping all other parameters constant; material size effects were probed by varying the tube wall thickness, t, from $200 \mathrm{~nm}$ to $635 \mathrm{~nm}$, at a constant relative density and grain size. In situ uniaxial compression experiments revealed an order of magnitude increase in yield stress and modulus in nanolattices with greater lattice angles, and a $150 \%$ increase in the yield strength without a concomitant change in modulus in thicker-walled nanolattices for fixed lattice angles. These results imply that independent control of structural and material size effects enables tunability of mechanical properties of three-dimensional architected metamaterials and highlight the importance of material, geometric, and microstructural effects in small-scale mechanics. [DOI: 10.1115/1.4030361]
\end{abstract}

Keywords: cellular solids, hierarchical materials, size effect, metamaterials

\section{Introduction}

Hierarchically designed cellular materials have been used for many decades as a method to create mechanically robust engineered structures-like the Eiffel Tower and the Garabit Viaduct. Introducing architectural elements enables creating structures that are lightweight, because they use a fraction of monolithic material with the same dimensions, and simultaneously strong since the architecture provides a way to more efficiently carry load in a structure. The mechanical performance of such architected solids on the macroscale is a function of the deformation mechanism and relative density of the structure, as well as of the constituent material properties, and this has been studied for both materials with cellular solid cores as well as bulk cellular solids [1-16]. Cellular solids can deform by either bending or stretching of the elements, which is dictated by the geometry of the lattice and its nodal connectivity, and this bending or stretching behavior defines the deformation mechanism of the cellular solid $[1,2,5,17,18]$. A three-dimensional structure must have a connectivity of $Z=6$ at the nodes to be rigid and a connectivity of $Z=12$ to be stretchingdominated; structures with $6 \leq Z<12$ are bending-dominated [18]. The structural deformation mechanism, determined by the nodal connectivity, directly impacts the modulus and yield strength of the overall structure [1,2]. The modulus and yield strength are also related to the structure's relative density, $\bar{\rho}$, which is defined as the volume of the lattice contained within a unit cell divided by the volume of the unit cell [2]. The yield strength and modulus of 3D open-cell bending-dominated structures, such as honeycombs or octahedron lattices, scale as

Contributed by the Applied Mechanics Division of ASME for publication in the Journal OF APPLIED Mechanics. Manuscript received November 20, 2014; final manuscript received April 9, 2015; published online June 3, 2015. Assoc. Editor: A. Amine Benzerga. $\sigma_{y}=0.3 \bar{\rho}^{1.5} \sigma_{y s}$ and $E=\bar{\rho}^{2} E_{s}$, where $\sigma_{y s}$ and $E_{s}$ are the yield strength and modulus of the constituent material [1,2]. For 3D stretching-dominated structures, such as the octet-truss lattice, the yield strength and modulus scale as $\sigma_{y}=0.3 \bar{\rho} \sigma_{y s}$ and $E=0.3 \bar{\rho} E_{s}$, which causes strength to decrease less rapidly than that of bending-dominated structures as relative density decreases $[1,5]$.

A theoretical upper limit for strength and modulus of cellular solids exists as a function of relative density because the mechanical properties of the lattice depend on the constituent material properties. All existing engineering materials with densities lower than $100 \mathrm{~kg} / \mathrm{m}^{3}$ fall below this theoretical maximum by an order of magnitude or more, leaving a prominent "white space" in the strength versus density material property space, shown in Fig. 1 [1]. When calculating this material property space, the strength and stiffness of the constituent materials are assumed to be constant so the deformation mechanism of the structure represents the only variable parameter. For macroscale cellular solids, this assumption is reasonable because yield strength is independent of size for materials at this length scale. Below the micron scale, many different classes of materials exhibit size effects, such as "smaller is stronger" in single crystalline metals [19-23], "smaller is weaker" in nanocrystalline metals [20,24-27], and "smaller is ductile" in metallic glasses and ceramics [20,28-31]. This renders the mechanical properties of materials at these dimensions different from bulk, and these properties are no longer constant. Creating hierarchical structural geometries with submicron dimensions, such as nanolattices, has been shown to provide a pathway to control and tune the strength and stiffness of cellular solids.

\section{Background}

2.1 Background: Cellular Solids. Relative densities of cellular solids can be modulated by several approaches, for example, 

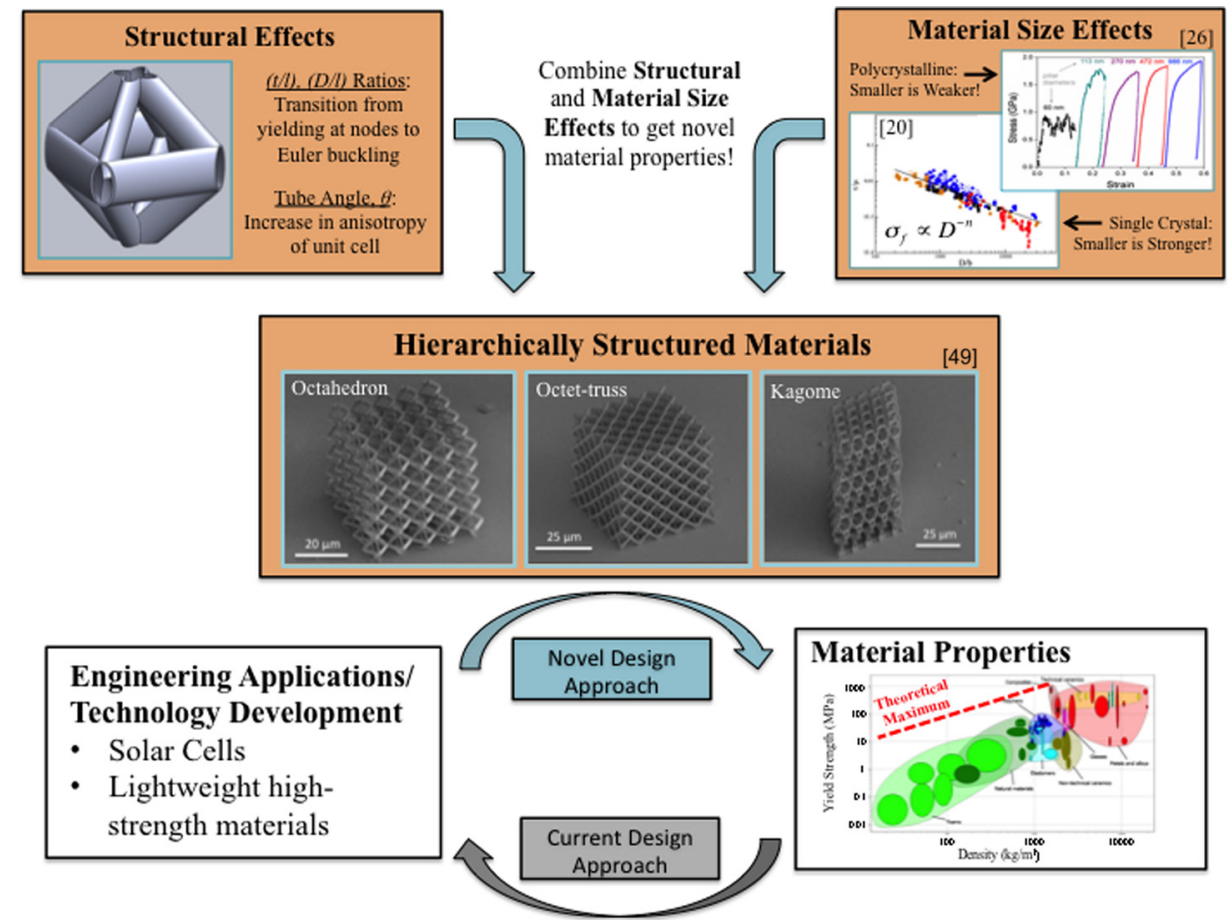

Fig. 1 Hierarchically structured materials combine structural and material size effects to enhance material properties and provide opportunities to create new materials that outperform current low-density materials. Material properties chart generated using CES SELECTOR (image courtesy of S. Das). (Figures reprinted with permission from Greer and De Hosson [20], Gu et al. [26], and Montemayor et al. [49]. Copyright 2011 by Elsevier Ltd. and 2012 by American Chemical Society, respectively.)

by using hollow tubes instead of solid rods within the same architecture. When hollow tubes are utilized in low-density cellular solids, structural effects can be activated by changes in the various ratios of geometric parameters that define the lattice tubes. While these structural effects do not fundamentally change the deformation mechanism of the entire lattice, deformation in the individual lattice tubes can be tuned by changing the geometry. For example, the compressive response of hollow thin-walled Ni-based microlattices demonstrated that its deformation behavior and recoverability depend on the geometric parameters of the lattice tubes, like the tube diameter-to-length ratio, $D / l$, and wall thickness-todiameter ratio, $t / l[9-11,28,32-34]$. Valdevit et al. proposed a model in which the deformation mechanism of the individual microlattice tubes transitions from Euler buckling to yielding of the constituent material at the nodes at a critical relative density, which is directly proportional to $(D / l)$ and $(t / l)$ [9]. This model assumes that all members of the unit cell carry equal force and that the maximum bending moment occurs at the nodes; the stress at the node is not taken into account [9]. The results of this analytic model compared favorably with those of the finite element method (FEM) simulations that account for the complex nodal geometry, where the latter also exhibited a critical relative density at which the deformation mode of the tubes transitioned from Euler buckling to yielding, albeit at lower overall stresses, and the transition occurred at a lower relative density [9]. Torrents et al. proposed a simple analytic model that predicts a critical $(t / D)$ ratio above which plastic deformation begins. This critical $(t / D)_{\text {crit }}$ is a function of the constituent material properties, tube angle, and maximum global strain of the microlattice [10]. Compression experiments showed that the microlattices fully recovered after $50 \%$ compressive strain for $(t / D)<(t / D)_{\text {crit }}$, which has been demonstrated for a variety of materials $[10,34]$. While the geometric ratios of the microlattices impact the deformation mechanism of the individual tubes, macroscopic parameters of the overall lattice, such as the lattice angle, also affect the mechanical response of the structure. Previous work defined the lattice angle to be the angle between the horizontal midplane of the unit cell and the lattice tubes, as shown in Fig. 2 [35,36]. Jacobsen et al. showed that strength and stiffness of solid polymer microlattices increased with higher lattice angle, as predicted by classical mechanics [35]. The calculated stiffness of the microlattices was found to be within $15 \%$ of the model proposed by Deshpande et al., which predicts that for a pyramidal core (stretching-dominated), the stiffness scales as $E=E_{s} \bar{\rho}(\sin \theta)^{4}[4,35]$. The microlattices presented in the work by Jacobsen et al. have a connectivity of $Z=8$, which does not rigorously satisfy the condition for a stretchingdominated structure and might explain the observed discrepancy

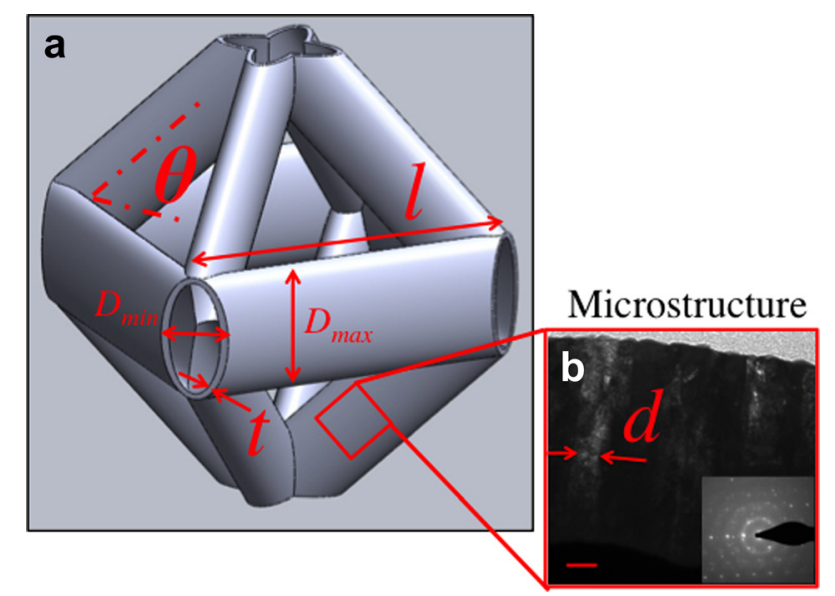

Fig. 2 (a) Schematic of relevant geometric parameters on a nanolattice unit cell and $(b)$ TEM image showing columnar grain structure of tubes with grain size on the order of $50 \mathrm{~nm}$ (TEM courtesy of $Z$. Aitken, scale bar $100 \mathrm{~nm}$ ) 
between the model and the experiments [35]. Previous work on microlattices demonstrates the importance of understanding structural effects that may potentially couple with material size effects in complex hollow-tube architectures and provides insight to utilizing structural effects in three-dimensional architected materials [4,9-11,28,32-35]. Valdevit et al. have shown in simulation that the analytical predictions for strength and stiffness where $\sigma_{y} \propto \bar{\rho}^{m}$ and $E \propto \bar{\rho}^{n}$ overpredict the strength of a hollow cellular solid by as much as an order of magnitude and the experimental results on hollow microlattices agree with the finite element simulations for a periodic cellular solid with hollow nodes [2,9]. Torrents et al. have shown for the microlattices that the strength follows the analytically predicted scaling relations, however, again a knock-down in strength is observed and this is attributed to the complex stress state at the hollow nodes under uniaxial loading [10]. Recently, Zheng et al. demonstrated the ability to create ceramic and ceramic-polymer composite structures with multiple geometries, on the same length scales as the microlattices, using a microstereolithographic process [6]. For stretching-dominated structures in the work by Zheng et al., the strength was found to scale as $\sigma_{y} \propto \bar{\rho}$ and the modulus as $E \propto \bar{\rho}$, for $10^{-4}<\bar{\rho}<10^{-1}$, which agrees with the classical theory proposed by Gibson and Ashby for stretching-dominated cellular solids [2,6]. Meza et al. have proposed a model to describe the local deformation behavior of the nanolattices by considering the compression of a single half unit cell; they found that the deformation of the tubes was governed by lateral torsional bending which is caused by minor geometric imperfections at the nodes and these results agree with the experimental data $[8,37]$. Meza et al. have also reported the mechanical behavior of stretching-dominated alumina nanolattices that were fabricated using two-photon lithography followed by deposition of a rigid coating and etching out of the polymer scaffold and found that the strength scales as $\sigma_{y} \propto \bar{\rho}^{1.76}$, the modulus scales as $E \propto \bar{\rho}^{1.61}$, and that the structures recovered after compression in excess of $50 \%$ [7]. The decrease in scaling for both strength and stiffness is attributed primarily to the complex stress state at the hollow nodes and the minor geometric imperfections of the nanolattices [7].

\subsection{Background: Material Size Effects}

2.2.1 Uniaxial Deformation. A variety of material size effects have been observed in nanoscale samples with different microstructures [19-27]. Single crystalline nanopillars exhibit a smaller is stronger size effect demonstrated through experiments, theory, and computations for face-centered-cubic (FCC), body-centeredcubic, hexagonal-close-packed metals [19-24,38-40]. For example, the yield strength of single crystalline FCC metals can be described by a power law dependence between strength and size, $\sigma \propto D^{-n}$, where $D$ is the pillar diameter and $n$ ranges between 0.5 and $0.7[19,20,23,24]$. Nanocrystalline metals with similar external dimensions exhibit the opposite effect on strength, with the emergence of a smaller is weaker size effect at a critical sample size-to-grain size ratio, $(D / d)$, unique to each material. For example, Pt nanopillars with $12 \mathrm{~nm}$ grains had a critical $(D / d)$ of $5-10$; in Ni nanopillars with $60 \mathrm{~nm}$ grains, the weakening occurred for $(D / d)$ between 15 and 30, and in polycrystalline $\mathrm{Cu}$ samples weakening was observed in experiments and simulations for $(D / d)<10$ [25-27]. Gu et al. proposed a model that describes how the yield strength of a nanocrystalline metal changes as a function of the diameter of a cylindrical sample relative to the material grain size [26]. This model assumes that only the grains in the outer shell interact with the free surface and the grains in the inner core of the sample are not affected by it [26]. The model predicts that for large $D / d$, the strength approaches the bulk strength, and for small $D / d$, the deformation is dominated by grain boundary sliding, which weakens the sample [26]. The following equation shows the relationship between the yield strength and the sample size-tograin size ratio, where $d$ is the grain size, $D$ is the sample size, $\sigma_{Y}^{s}$ is the stress required to initiate grain boundary sliding, and $\sigma_{y \text { (bulk) }}$ is the strength of the bulk material with the same microstructure [26]

$$
\frac{\sigma_{n(\mathrm{Au})}}{\sigma_{y(\text { bulk })}}=\left(1-\frac{d}{D}\right)^{2}+\frac{\sigma_{Y}^{s}}{\sigma_{y(\text { bulk })}}\left[1-\left(1-\frac{d}{D}\right)^{2}\right]
$$

2.2.2 Beyond Nanopillars: Deformation Under Complex Stress States. Hierarchical architected structures allow opportunities for material size effects to be utilized because the novel material properties present at the submicron length scales can be proliferated onto a larger structure. Hodge et al. showed that a stochastic nanocrystalline Au nanoporous open-cell foam exhibits a smaller is stronger size effect as the ligament size decreases from $900 \mathrm{~nm}$ to $10 \mathrm{~nm}$; these foams outperform the expected strength of a cellular solid with a relative density of $\sim 0.24-0.32$ according to the scaling law, where $\sigma_{y}=0.3 \bar{\rho}^{1.5} \sigma_{y s}$ [16]. Larger ligament sizes lead to a reduction in strength, with the precise mechanism still being a matter of ongoing discussion due to the complexity of the interplay between microstructure and free surfaces in the nanoporous foams [16].

In contrast to the stochastic nature of the Au foams, the microlattices have an ordered and controllable geometry, which allows the exploitation of the smaller is ductile material size effect for some material systems [28]. For example, Rys et al. showed that the compressive strength of $\mathrm{NiP}$ metallic glass microlattices with relative densities of $10^{-4}$ is an order of magnitude higher than that of nanocrystalline NiP microlattices with the same geometry [28]. That work also demonstrated that these metallic glass hollow-tube microtrusses with $150 \mathrm{~nm}$-thick tube walls deformed in a ductile fashion, in contrast to their thicker-walled counterparts, which undergo catastrophic failure upon loading. Such a transition in deformation mode from brittle to ductile has been reported for nanosized metallic glasses and has been attributed to the energetic balance between shear band propagation and homogeneous deformation [20,28-31]. The authors also showed that the scaling of yield strength with density decreases from $\rho^{2.3}$ to $\rho^{1.4}$ when the wall thickness falls below $150 \mathrm{~nm}$ [28]. Gu et al. have shown that material size effects can also be exploited to enhance the strength and stiffness of solid tube lattices with relative densities between $40 \%$ and $80 \%$ with single crystalline regions within the ligaments [41]. For lattices with relative densities above $50 \%$, Gu et al. observed a strength 1.8 times that of the bulk $\mathrm{Cu}$ as a result of the smaller is stronger size effect [41]. This work aims to show that nanolattices can exploit both structural and material size effects that have been observed in previous work independently to tune the strength and stiffness of an ordered cellular solid of a given relative density.

\section{Experimental Methods}

Nanolattices in this work were fabricated using two-photon lithography with a $780 \mathrm{~nm}$ femtosecond pulsed laser (Nanoscribe $\mathrm{GmbH}$, Eggenstein-Leopoldshafen, Germany) [42-48]. This technique involves the constructive interference of two photons within a three-dimensional voxel, which provides a sufficient amount of energy to cross-link the photoresist within the voxel [42]. The photoresist is placed on a substrate, which is then mounted on a stage that can move in all directions with a resolution of $\pm 10 \mathrm{~nm}$ and a maximum displacement of $300 \mu \mathrm{m}$. The cross-linked polymer sample can be of any geometry with features down to $150 \mathrm{~nm}$ $[44,48]$. The cross section of the tubes in the nanolattices is elliptical, which is an artifact of the two-photon fabrication process, and the ellipticity of the individual tubes changes as a function of the lattice angle. The samples were developed in propylene glycol monomethyl ether acetate followed by an isopropyl alcohol rinse to isolate the polymer nanolattices, which were then sputtered with approximately $200-635 \mathrm{~nm}$ of columnar grained Au at 3 $\mathrm{mTorr}$ and $50 \mathrm{~W}$. The internal polymer scaffold was then exposed using a focused ion beam and removed using an $\mathrm{O}_{2}$ plasma etch [49]. 
The transmission electron spectroscopy (TEM) image of the tube walls shown in Fig. 2 confirms that the sputtered Au has a columnar structure, with the grains oriented orthogonally to the length of the tubes, which is consistent with the typical columnar grains on flat substrates [50-52]. The size of the grains ranged from 50 to $100 \mathrm{~nm}$ when measured using a cross section parallel to the length of the columnar grains and $25-50 \mathrm{~nm}$ when measured from a cross section perpendicular to the length of the columnar grains (Fig. 2(b)). All samples were designed to have octahedron geometry and a constant relative density, i.e., structures with thicker tube walls had larger unit cells. A geometric model was developed in SOLIDWORKS and was used to predict the geometric parameters needed such that the relative density would be $\bar{\rho} \approx 0.02-0.04$ for all samples. After the samples were fabricated, the geometric parameters were measured using scanning electron microscopy (SEM) and these values were used in the SOLIDWORKS model to calculate the relative densities of the fabricated nanolattices, which was found to be $\bar{\rho}=0.05 \pm 0.01$. The mean and standard deviation for the relative density were calculated by averaging the relative densities of all fabricated samples, which was determined using SEM images for each fabricated sample. Thicker tube walls caused greater nonuniformity of the coating, especially near unit cells along the edge of the nanolattice, and this is shown in Fig. 3. For all samples, the wall thicknesses were measured from SEM images at multiple points along the height, width, and depth of the compressed regions of the nanolattice.

To exploit structural effects, we fabricated and tested octahedron nanolattices with angles of $30 \mathrm{deg}, 45 \mathrm{deg}$, and $60 \mathrm{deg}$, and a constant relative density of $\bar{\rho}=0.05 \pm 0.01$. The lattice angle is defined in this work to be the angle between the horizontal midplane of the unit cell and the lattice tubes, consistent with previous work, and is shown in Fig. 2. The wall thicknesses in these samples were $t=336 \pm 58 \mathrm{~nm}$. The wall thicknesses presented in this work are in the regime where material size effects have been observed for nanopillar geometries, which are discussed in Sec. 2.2. Material size effects were probed by varying the wall thickness for the octahedron geometry nanolattices with constant relative density of $\bar{\rho}=0.05 \pm 0.01$ and a fixed angle of $45 \mathrm{deg}$; the same experiment was repeated for lattices with a fixed angle of $60 \mathrm{deg}$. Mechanical properties of the nanolattices were obtained by conducting uniaxial compression experiments in an in situ nanomechanical instrument (InSEM, Nanomechanics, Inc., Oak Ridge, TN). Samples were compressed to strains of $40-60 \%$ at a constant prescribed strain rate of $\dot{\varepsilon}=10^{-4} \mathrm{~s}^{-1}$ or lower and load-displacement data were collected. A diamond flat punch with a diameter of $170 \mu \mathrm{m}$ was used to compress the nanolattices. The stress was calculated according to $\sigma=F / A$, where $F$ is the measured load, and $A$ is the total cross-sectional area of the nanolattice, and the yield stress was calculated using the $0.2 \%$ offset method. The modulus was calculated using the slope from the elastic loading regime and was calculated using $\mathcal{E}=\Delta H / H$, where $H$ is the height of the nanolattice, and $\Delta H$ is the measured displacement. The unloading slope of the stress-strain curve was not used to determine the modulus of the nanolattice, since the structures deform plastically and the unloading slope would give the modulus of the densified structure; initial regions where the sample may not have fully been in contact with the punch were excluded from the modulus calculation, though these were minimal across the data.

\section{Results}

4.1 Results: Structural Effects. To determine the effect of geometry on the mechanical properties of the nanolattices, experiments were performed on octahedron unit cells with angles of $30 \mathrm{deg}, 45 \mathrm{deg}$, and $60 \mathrm{deg}$. Figure 4 shows SEM images of the nanolattices before and after compression, as well as stress-strain data for a representative sample for each lattice angle. A circle on the stress-strain curves denotes the yield stress for each of the nanolattices shown in Fig. 4. Four samples were fabricated for each lattice angle and the $0.2 \%$ yield stress and modulus were found by averaging over all samples for each angle, and the data are shown in Fig. 5. These plots show that higher angle in the unit cells leads to higher yield strength and stiffness. As the angle changes from $30 \mathrm{deg}$ to $60 \mathrm{deg}$, the yield stress increases from $161.3 \pm 18.5 \mathrm{kPa}$ to $858.2 \pm 68.4 \mathrm{kPa}$, and the stiffness increases from $8.42 \pm 0.7 \mathrm{MPa}$ to $85.2 \pm 15.0 \mathrm{MPa}$. The data also show that the yield stress was reached at lower strains at higher lattice angles.

4.2 Results: Material Size Effects. To examine the ability of nanolattices to exploit material size effects, uniaxial compression experiments were performed on the octahedral nanolattices with two different fixed lattice angles, $45 \mathrm{deg}$ or $60 \mathrm{deg}$, and the wall thickness varied from $t=200-635 \mathrm{~nm}$. Figure 6 shows the images

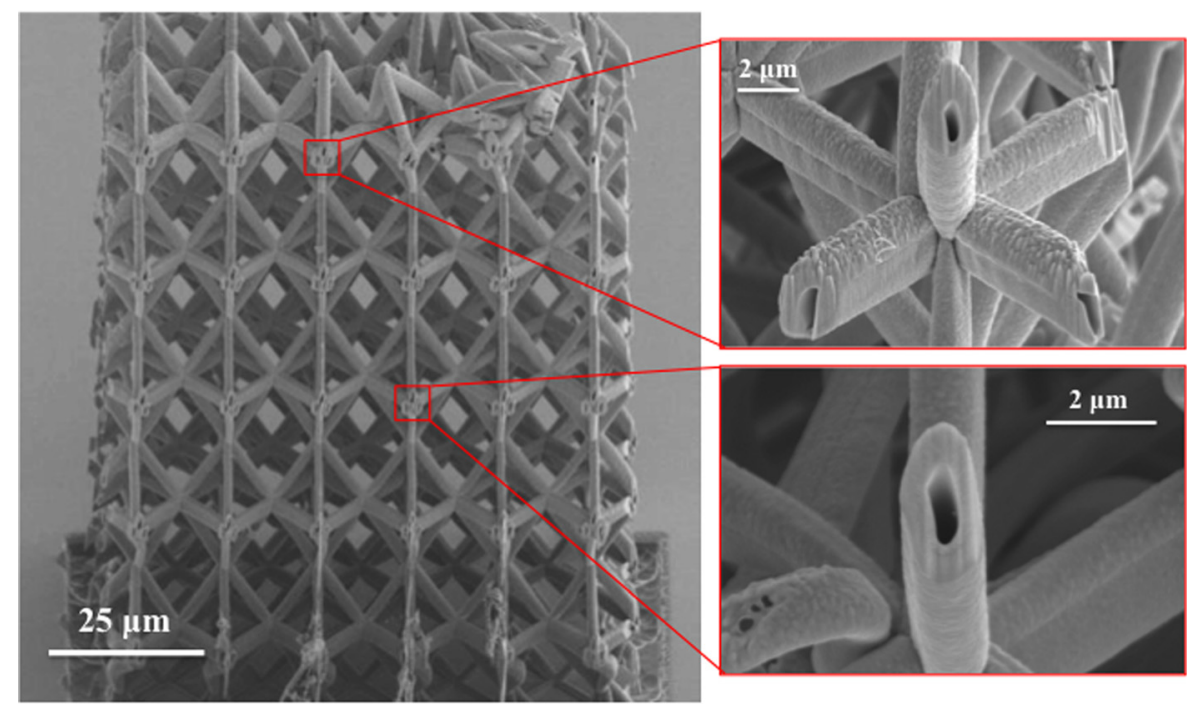

Fig. 3 Representative images of the Au coating in a 60 deg nanolattice with a wall thickness of $\sim 661 \mathrm{~nm}$. In the center region, the nanolattice walls are conformal; however, the coating is not conformal near the edges of the lattice as a result of the anisotropy of the sputtering process. 

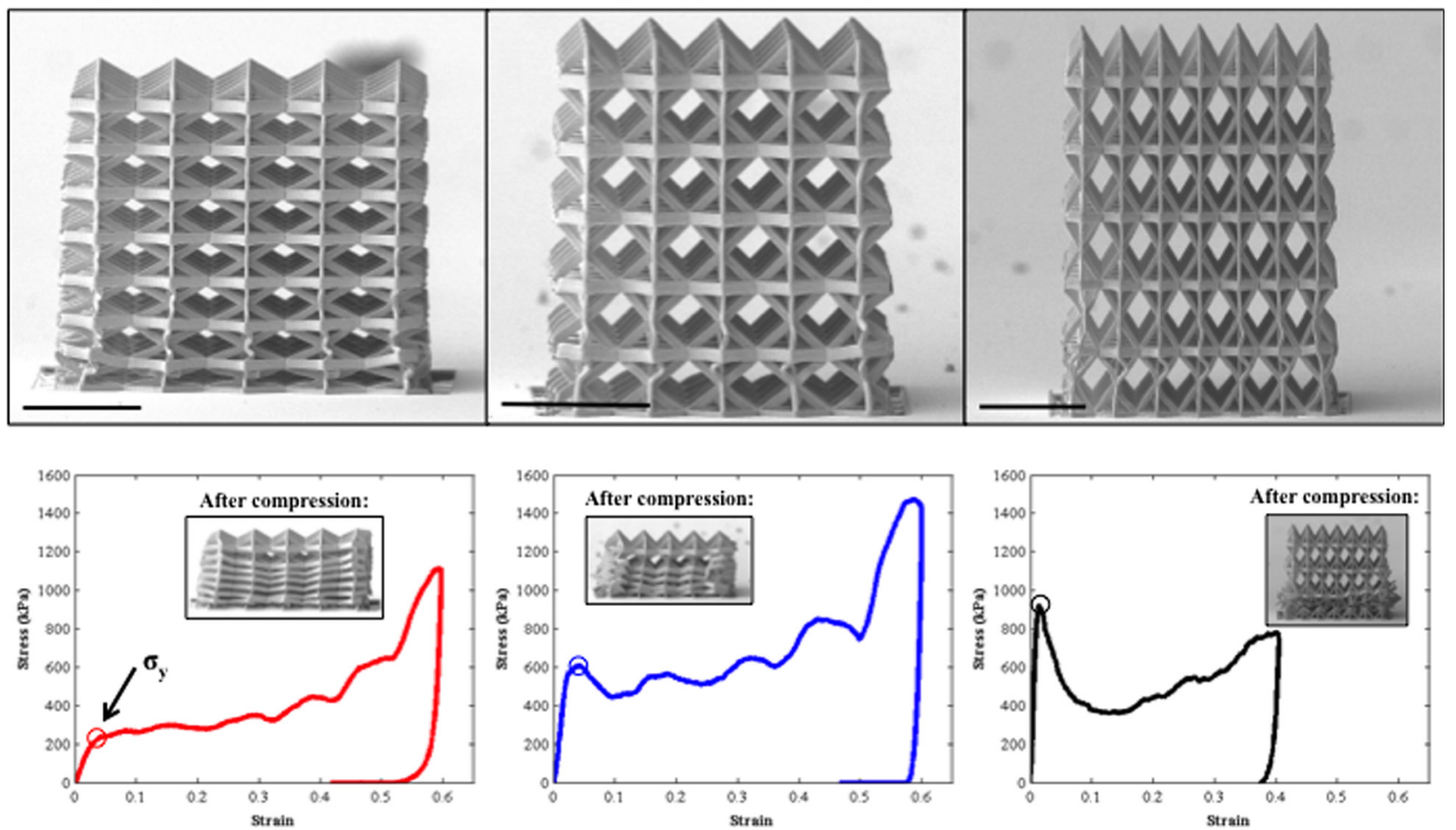

Fig. 4 SEM image of octahedron nanolattices with lattice angles from $30 \mathrm{deg}$ to $60 \mathrm{deg}$, as well as representative stress-strain curve for each sample with an open circle showing the $0.2 \%$ yield stress of the structure. For the 30 deg lattice, it should be noted that the error bars are included but are small enough to be obscured by the data point itself (scale bar denotes $30 \mu \mathrm{m})$.

of 45 deg nanolattices before and after compression, as well as the representative stress-strain data for each structure. For the $45 \mathrm{deg}$ nanolattices, the yield stress was calculated by taking the average of four samples for each of the $t=200 \mathrm{~nm}$ and $327 \mathrm{~nm}$ cases and one sample for $t=635 \mathrm{~nm}$ case. The data indicate that for the 45 deg nanolattices, the yield stress increased by a factor of 2.1 , or by $\sim 120 \%$, as $t$ increased from $200 \mathrm{~nm}$ to $327 \mathrm{~nm}$ and by $\sim 13 \%$ as $t$ increased from $327 \mathrm{~nm}$ to $635 \mathrm{~nm}$. The $0.2 \%$ yield stress increased with $t$ but occurred at approximately the same strain for all 45 deg samples. Yield stresses were also calculated for nanolattices with a lattice angle of $60 \mathrm{deg}$ for two samples with $t=325$ $\mathrm{nm}$ and one sample with $t=661 \mathrm{~nm}$. The $60 \mathrm{deg}$ nanolattices appeared to be $\sim 58 \%$ stronger than the 45 deg nanolattice for all wall thicknesses, as shown in Fig. 7, which is consistent with the results from literature [35]. The yield stress of the 60 deg nanolattices increased by $\sim 14 \%$ as $t$ varied from $325 \mathrm{~nm}$ to $661 \mathrm{~nm}$. Yielding occurred at approximately the same strain of $1.2 \%$ for all $60 \mathrm{deg}$ samples, which is lower than $1.7 \%$ yield strain in the 45 deg samples. The large error bars for tests on samples with the thickest walls, shown in Fig. 7, are likely a result of the anisotropy introduced by the sputtering process. All deformation initiates at the base of the nanolattice structures, where the coatings are more conformal compared to the top layer on unit cells, so the mean is a more representative wall thickness for the area of the nanolattice that deforms during the experiments.

\section{Discussion}

Building upon an analytic model proposed by Valdevit et al. to calculate the stress required to initiate Euler buckling or yielding along the tubes for a microlattice with hollow circular tubes, we account for the hollow elliptical tubes in the octahedron lattice geometry presented in this work $[9,53]$. Since changes in the geometric parameters of the nanolattices, such as wall thickness or tube diameter can lead to structural effects, the initial deformation of the tubes must be consistent across all samples with varying geometric parameters such that lattice angle and material size effects can be isolated. For example, under an applied load the lattice tube can either undergo Euler buckling or yielding of the constituent material as the tubes are compressed; as the geometric parameters of tubes, such as the length and wall thickness, are varied it may be possible to initial buckling prior to yielding of the constituent material. The critical values to initiate Euler buckling and yielding of the elliptical tubes for the nanolattice geometry are shown in the following equations (supplemental derivations are available under the "Supplemental Data" tab for this paper on the ASME Digital Collection):

$$
\begin{gathered}
\sigma_{\text {buckling }}=8 \pi^{3} E \cos ^{2} \theta \sin \theta\left(\frac{D_{\text {min }}}{l}\right)^{2}\left(\frac{D_{\min }+3 D_{\text {max }}}{l}\right)\left(\frac{t}{l}\right) \\
\sigma_{\text {yield }}=4 \pi \sin \theta\left(\frac{t}{l}\right)\left(\frac{D_{\min }+D_{\text {max }}}{l}\right) \sigma_{y(\mathrm{Au})}
\end{gathered}
$$

The geometric parameters $\left(D_{\min }, D_{\max }, t\right.$, and $\left.l\right)$ used to calculate the critical values to initiate Euler buckling or yielding of the nanolattice tubes are schematically defined in Fig. 2(a) and tabulated for each sample in Table 1. The yield stress of the Au, $\sigma_{y(\mathrm{Au})}$, in Eq. (3) was measured via nano-indentation into $2.17 \mu \mathrm{m}$ thick film on a glass substrate to a depth of $100-200 \mathrm{~nm}$ to be $583 \pm 41 \mathrm{MPa}$, and the indentation modulus to be $E=95 \pm 4 \mathrm{GPa}$, both calculated using the Oliver-Pharr method [54]. The stresses required to initiate either yielding or Euler buckling in the $\mathrm{Au}$ nanolattices were calculated using Eqs. (2) and (3) and are shown in Table 1. The experiments presented in this work consider structures of constant relative density and lattice type, therefore the geometric parameters $\left(D_{\min }, D_{\max }, t\right.$, and $\left.l\right)$ of the hollow tubes must change with the wall thickness, $t$. As a result, all samples presented in this work have varying values of $D_{\min }, D_{\max }, t$, and $l$, which are shown in Table 1 along with the critical stress to initiate 

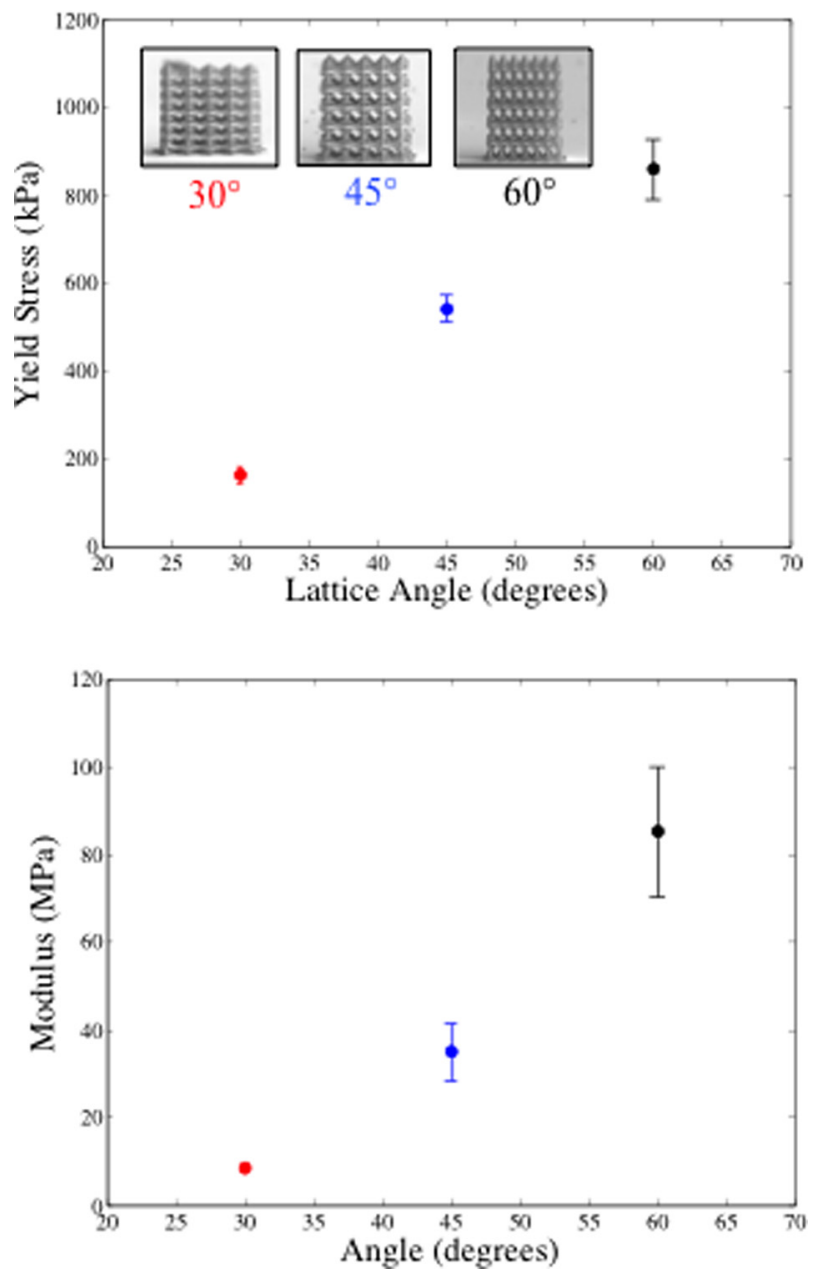

Fig. 5 Calculated yield stress and modulus values for lattices with angles ranging from $30 \mathrm{deg}$ to $60 \mathrm{deg}$. The error bars were calculated by using the standard deviation of the data for yield stress and modulus (note: $t=352 \pm 87 \mathrm{~nm}$ for all samples).

Euler buckling and yielding. We found that for all nanolattices in this work, the threshold stress for yielding was in the range between 164 and $290 \mathrm{MPa}$, which is more than an order of magnitude lower than that required for Euler buckling (3.0-12.5 GPa). Potential errors in the magnitude of the critical stress values may be introduced if size effects cause the value of $\sigma_{y(\mathrm{Au})}$ to vary as the wall thickness increases, however, based on previous work an order of magnitude change in the yield strength of the $\mathrm{Au}$ is not expected due to size effects [24,26]. This implies that all samples will fail by yielding upon compression and that any geometric changes required to keep relative density constant do not change the fundamental deformation behavior of the nanolattice tubes. We observed that the initiation of yielding always occurred at the hollow nodes likely because of the substantial local stress. The dominance of nodal deformation within the structure renders minor nonuniformities within the wall thickness relatively inconsequential for mechanical behavior.

We found that the stresses predicted for the onset of yielding by Eq. (3), within the range of 164-290 MPa, did not match the experimental data; which ranged from 161 to $976 \mathrm{kPa}$ (see Table 2). This discrepancy is likely caused by the fundamental limitations in the simple mechanics model in its inability to capture the complex stress state within the hollow structure. For example, the model accounts for buckling or yielding along the thin-shell hollow tubes with elliptical cross sections but does not account for the complex geometry and stress state at the nodes where the tubes connect. The nanolattices experience higher stress concentrations at the nodes because of the small radius of curvature where tubes converge induces the onset of yielding at the node instead of along the length of the tube. Our experimental observations and the analytic calculations confirmed that the deformation was consistently initiated by yielding at the nodes for all samples in this study. Valdevit et al. found that the analytic calculations for microlattices were approximately 1-2 orders of magnitude higher than those predicted by the FEM and than was observed experimentally, which is consistent with the observations in this work [9]. A deviation of 1-2 orders of magnitude in strength has also been observed experimentally in micro- and nanolattices, and the deviation from the analytically predicted strength and stiffness in this work is consistent with the existing literature $[7,10]$. The tight distribution in the yield stresses in our experiments, shown in Table 2, implies that structural deformation in all samples likely occurred via yielding rather than buckling because this type of deformation is generally robust against defects. Buckling is an example of instability phenomena where minor defects in the nanolattice geometry could significantly affect the critical stress required to initiate buckling and produce a wider spread in the data, which is not observed in these experiments [53]. The low standard deviation observed across all data in this work is consistent with the prediction that failure of the tubes initiates by yielding based on the model proposed by Valdevit [53]. Experimental observations revealed that the sample first began to rotate and deform at the nodes prior to bending of the tubes, which may be induced by imperfections in the nanolattices.

5.1 Discussion: Structural Effects. We found that the yield stress and modulus of the structures increase with the lattice angle of the unit cell (Fig. 5). For the 30 deg case, the horizontal members of the unit cell carry more load in tension than would be experienced by the horizontal members in the $60 \mathrm{deg}$ samples when a uniaxial force is applied to a sample based on a simple force balance. Alternatively, the vertical members of the $60 \mathrm{deg}$ unit cell carry more load in compression than those of the $30 \mathrm{deg}$ unit cell under the same uniaxial loading conditions. For beams loaded in tension, a slight prebend or misalignment has a much less significant effect on the mechanical behavior compared to a beam loaded in compression with an equivalent prebend or misalignment. As a result of the distribution of load across the unit cell as the lattice angle varies, it is expected that larger standard deviation would be observed in the 60 deg samples and this is observed in the data.

The model proposed by Deshpande et al., which relates the modulus of a stretching-dominated pyramidal core, can be extended to this work since the pyramidal core is half of the octahedron unit cell [4]. Though the octahedron unit cell is a stretching-dominated structure, the octahedron nanolattices behave as bending-dominated structures due to rotation between and within unit cells as the lattice is compressed. This rotation is initiated by the initial imperfections in the unit cell layer closest to the substrate, which is a result of the polymer contraction that occurs during the development process. Minor imperfections and misalignments within the nodes of the octahedron nanolattices have been reported to induce lateral torsional bending in the nanolattice struts, which also contributes to the bending-dominated behavior observed in this work [8,37]. The minor imperfections in the nanolattices, which are caused by the position accuracy of the two-photon fabrication system as well as the contraction of the polymer upon cross-linking, are an order of magnitude smaller than the unit cell size and account for the deformation mode of lateral torsional bending, as shown in existing literature [8,37]. The trend in the data follows a modified form of the modulus relation proposed by Deshpande et al., shown in Eq. (4) and Fig. 8(a), to include the correct scaling for a bending-dominated structure

$$
E=E_{s} \bar{\rho}^{m}(\sin \theta)^{4}
$$

with $m=2$ which is consistent with the theory classical theory for bending-dominated structures, where $E \alpha \bar{\rho}^{2}[1,2]$. Material size 


$$
t=200 \pm 53 \mathrm{~nm} \quad t=327 \pm 48 \mathrm{~nm} \quad t=635 \pm 162 \mathrm{~nm}
$$
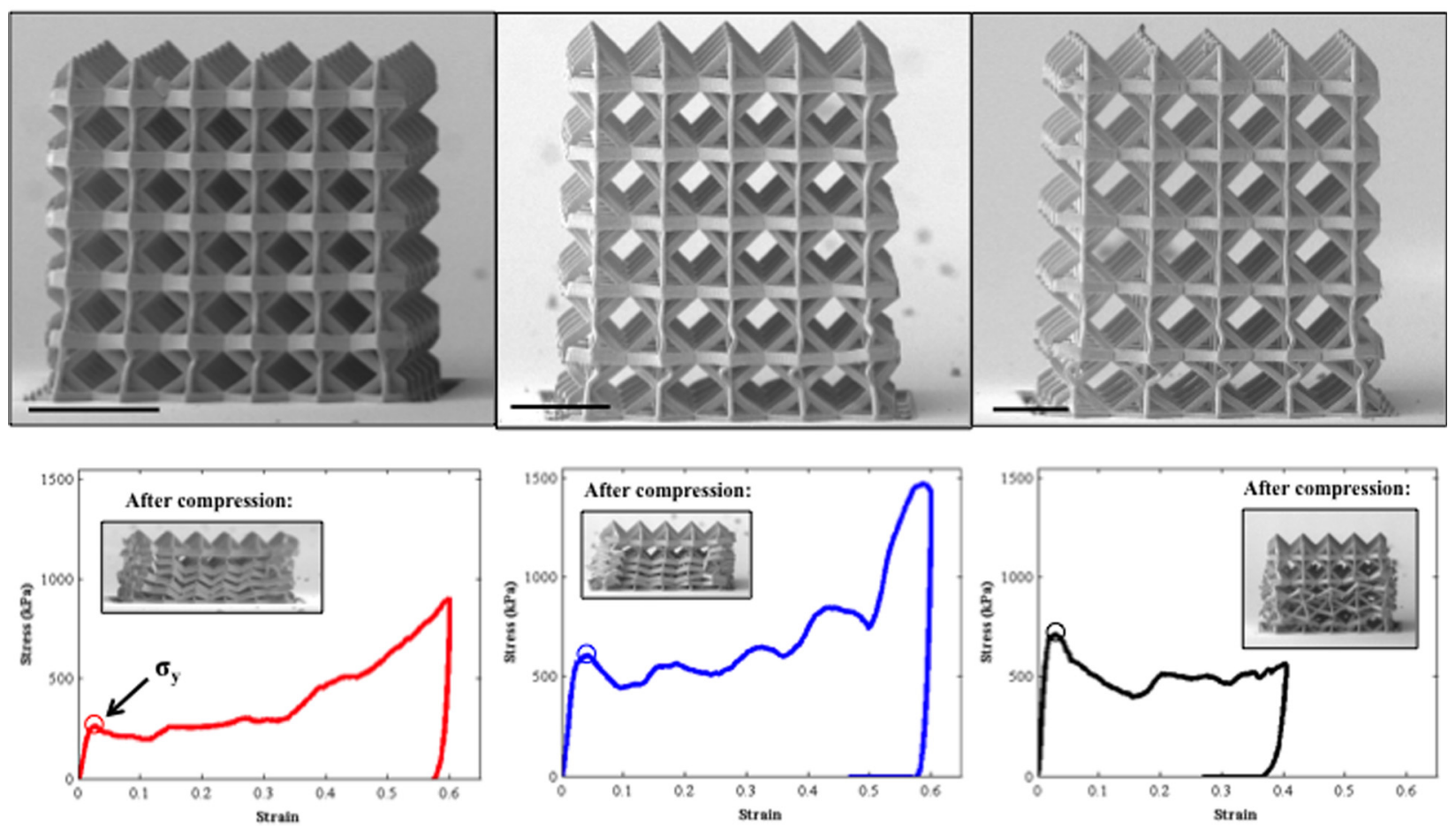

Fig. 6 SEM image of $45 \mathrm{deg}$ octahedron nanolattices with $t$ ranging from 200 to $635 \mathrm{~nm}$, as well as representative stress-strain curve for each sample with an open circle showing the $0.2 \%$ yield stress of the structure (scale bar denotes $20 \mu \mathrm{m})$

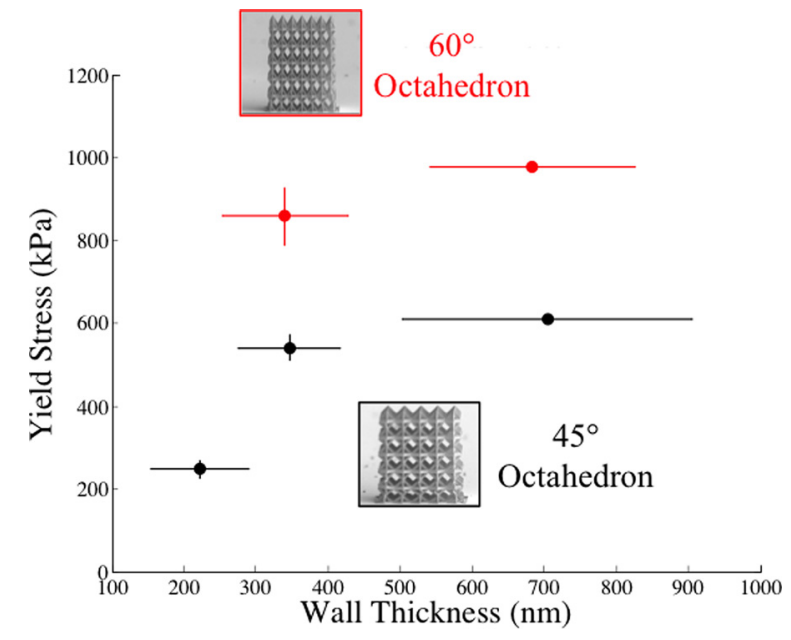

Fig. 7 Calculated yield stress for both 45 deg and 60 deg octahedron nanolattices. The yield strength increases as $(t / d)$ increases due to the material size effect. effects do not affect the modulus at these length scales, so $E_{s} \sim E$ of $\mathrm{Au}$ measured using nano-indentation. For bending-dominated structures where $E \alpha \bar{\rho}^{m}$ with $m \sim 2$, the data also scale with $\sin ^{4}(\theta)$ as predicted by literature; $m$ of $\sim 2.15$ appears to provide a better fit to experimental data, however, to do a proper regression model to determine the best fit value for $m$, a wider range of lattice angles must be tested [35]. The discrepancy in the relative density exponent is likely an artifact of the hollow nodes and it is expected that the nanolattices in this work would be less stiff than a cellular structure with solid nodes, which would scale with $m=2$.

A similar analysis was done for the yield strength and compared to the predicted value for a stretching-dominated structure predicted by Deshpande and Fleck [4]. A stretching-dominated pyramidal structure was predicted to scale as $\sigma=\sigma_{s} \bar{\rho}(\sin \theta)^{2}$, however, a bending-dominated geometry, such as the octahedron nanolattices in this work, should scale as $\sigma \alpha \bar{\rho}^{1.5}[1,2,4]$. Modifying the model proposed by Deshpande et al. to account for variation in the relative density exponent is given in the following equation:

Table 1 Relevant average geometric parameters, as well as threshold stress values for both yielding and Euler buckling, for all samples

\begin{tabular}{|c|c|c|c|c|c|c|c|c|}
\hline Geometry & $\begin{array}{l}\text { Angle } \\
\text { (deg) }\end{array}$ & $\begin{array}{c}\text { Thickness, } \\
t(\mathrm{~nm})\end{array}$ & $\begin{array}{l}\text { Major axis, } \\
D_{\max }(\mu \mathrm{m})\end{array}$ & $\begin{array}{l}\text { Minor axis, } \\
D_{\min }(\mu \mathrm{m})\end{array}$ & $\begin{array}{l}\text { Tube length, } \\
\quad l(\mu \mathrm{m})\end{array}$ & $\begin{array}{l}\text { Thickness-to-length } \\
\text { ratio, } t / l\end{array}$ & $\begin{array}{l}\sigma_{\text {yield }} \\
(\mathrm{MPa})\end{array}$ & $\begin{array}{r}\sigma_{\text {bucking }} \\
(\mathrm{GPa})\end{array}$ \\
\hline \multirow[t]{6}{*}{ Octahedron, $\bar{\rho}=0.05 \pm 0.01$} & 30 & 357 & 2.68 & 1.31 & 7.97 & 0.05 & 164 & 12.5 \\
\hline & 45 & 200 & 2.39 & 0.80 & 4.77 & 0.05 & 290 & 16.4 \\
\hline & & 327 & 2.76 & 1.17 & 7.96 & 0.04 & 210 & 8.8 \\
\hline & & 635 & 3.21 & 1.65 & 11.12 & 0.06 & 259 & 10.6 \\
\hline & 60 & 324 & 2.71 & 1.18 & 9.71 & 0.04 & 170 & 2.4 \\
\hline & & 661 & 3.14 & 1.53 & 13.07 & 0.05 & 229 & 3.0 \\
\hline
\end{tabular}


Table 2 Average measured and predicted yield stresses and modulus for the fabricated samples

\begin{tabular}{|c|c|c|c|c|c|}
\hline $\begin{array}{l}\text { Lattice } \\
\text { angle (deg) }\end{array}$ & $\begin{array}{l}\text { Wall thickness } \\
(\mathrm{nm})\end{array}$ & $\begin{array}{l}\text { Measured yield } \\
\text { stress }(\mathrm{kPa})\end{array}$ & $\begin{array}{c}\text { Analytic scaling } \\
\text { law: } \sigma=0.3 \sigma_{s} \rho^{1.5}(\mathrm{MPa})\end{array}$ & $\begin{array}{c}\text { Measured modulus } \\
(\mathrm{MPa})\end{array}$ & $\begin{array}{l}\text { Analytic scaling law: } \\
E=E_{s} \rho^{2}(\mathrm{GPa})\end{array}$ \\
\hline \multirow[t]{3}{*}{45} & 200 & 246 & 1.96 & 15.8 & 4.75 \\
\hline & 327 & 540 & 1.96 & 34.9 & 4.75 \\
\hline & 635 & 610 & 1.96 & 56.0 & 4.75 \\
\hline \multirow[t]{2}{*}{60} & 324 & 858 & 1.96 & 85.2 & 4.75 \\
\hline & 661 & 976 & 1.96 & 77.0 & 4.75 \\
\hline
\end{tabular}

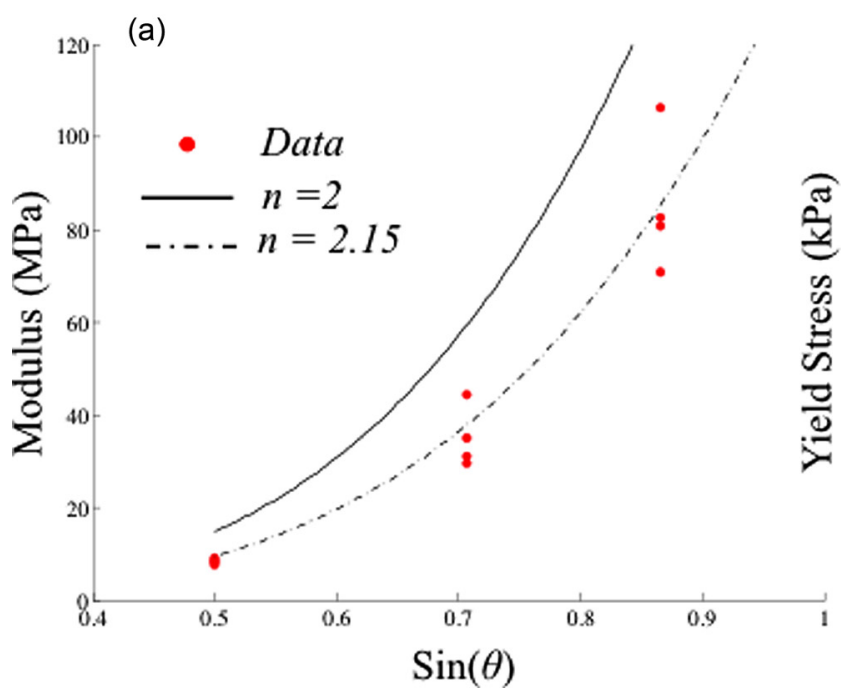

(b)

Fig. 8 (a) Modulus versus sine of lattice angle according to Eq. (4) and (b) yield stress versus sine of lattice angle according to Eq. (5)

$$
\sigma=\sigma_{s} \bar{\rho}^{n}(\sin \theta)^{2}
$$

Equation (5) captures the trend seen in calculated yield stresses for $n=1.5$, shown in Fig. $8(b)$. The calculated yield stress deviates from that predicted using Eq. (5) by approximately an order of magnitude or more as the lattice angle increases. This is likely because of the hollow nodes and small imperfections, such as minor misalignment at the nodes or prebend in the unit cells connected to the substrate, all of which may emerge as a result of the fabrication process. The behavior or the lattice is more sensitive to imperfections as the lattice angle increases, as shown by the larger spread in the data at higher angles, and we attribute to the increasing deviation from the predicted value according to Eq. (5) to this imperfection sensitivity at higher lattice angles. Material size effects alter the strength of the constituent material at the length scale present in the wall thickness of the nanolattices, however, a model to describe this change in constituent properties for this geometry and microstructure is outside of the scope of this work. The $\sigma_{s} \bar{\rho}^{n}$ term acts as a constant in the yield stress $\sim f(\sin (\theta))$ relation therefore the value of $n$ cannot be determined until the microstructure and material size effects present in the nanolattices are further characterized. The deviation in the $m$ and $n$ values from that which is expected of a bending-dominated structure ( $m=2$ and $n=1.5$ ) is likely due to the complex stress state at the hollow nodes that initiates failure well below analytically predicted values. The scaling laws for cellular solids assume pin-jointed structures, which is not the case for the fabricated samples presented in this work, therefore it is expected that the $m$ and $n$ values would deviate from those predicted using analytic theory $[1,2]$. Additionally, deviations of up to an order of magnitude between experiments, models, and analytic predictions for strength have been observed in previous work [9].

5.2 Discussion: Material Size Effects. The yield strength of the Au tube walls is expected to be lower than that of the same

material in monolithic form because the nanolattices in this work have wall thicknesses in the range where the reduced sample dimensions can lead to weakening in metals with nanometer-sized grains [19-27]. The applicability of the model proposed by $\mathrm{Gu}$ et al. in Eq. (1) to the nanolattice system is limited because the tube walls of the nanolattices are effectively a freestanding thin film in which the grains are confined laterally, although multiple grains may also span the wall thickness. The thickness of the nanolattice walls, $t$, and the microstructure of constituent material are relevant parameters for predicting the strength of the hollow $\mathrm{Au}$ tubes. The nanolattices in this work represent an

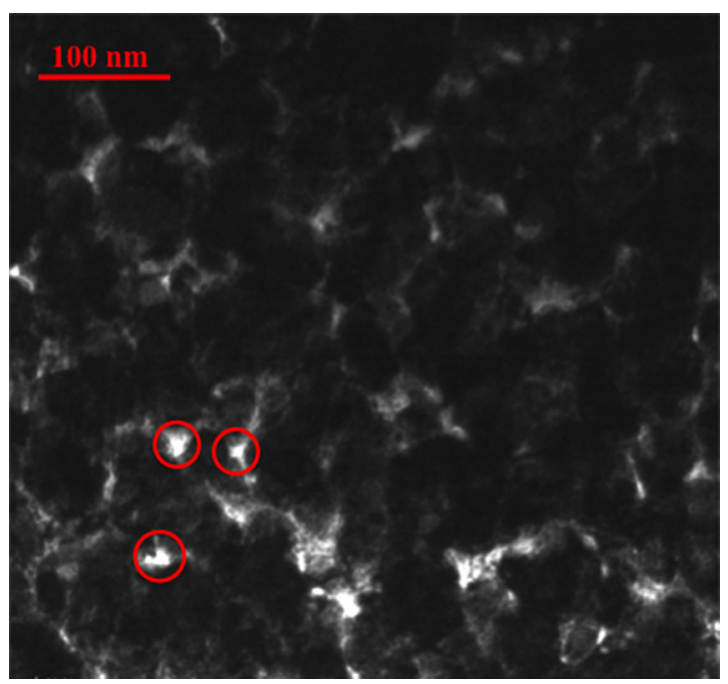

Fig. 9 TEM bright field image showing pores between grains as viewed through a section of a nanolattice wall, where the wall thickness is $\sim 200 \mathrm{~nm}$ 

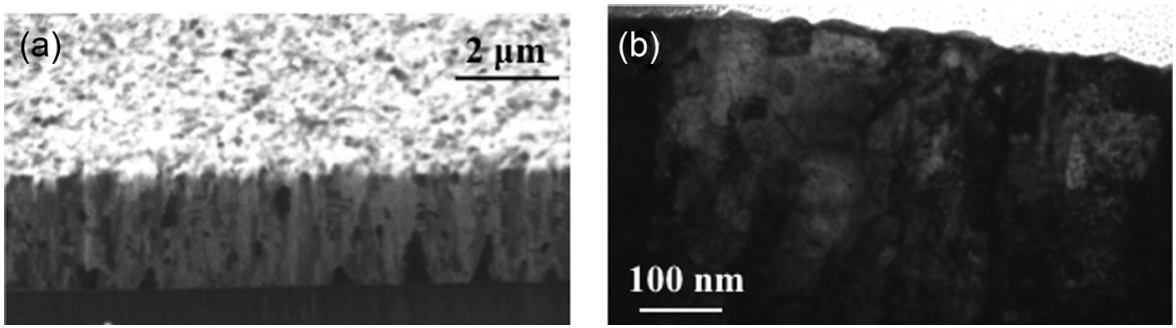

Fig. 10 (a) lon channeling (sample at 52 deg tilt) and (b) TEM images of a $\sim 2 \mu \mathrm{m}$ thin $n$-Au film showing columnar grain structure with multiple grains spanning the film thickness

interconnected system of such tubes and demonstrate that the material size effects observed in a simple nanopillar geometry can be exploited to control and tune the mechanical properties of the entire structure. Though a model to describe the material size effect is outside the scope of this work, any geometric parameters that potentially cause structural effects or changes in relative density are held constant and the structures are self-similar as wall thickness increases, so we attribute the observed lower yield stress to changes in the microstructure of the constituent material as the wall thickness dimension decreases. Table 2 shows the predicted yield stress and modulus of a structure with a fixed geometry and relative density, assuming the constituent material properties are constant, as well as the measured yield stress and modulus. The discrepancy in the measured and analytically predicted values is 1-2 orders of magnitude, which may be attributed to the complex stress state at the hollow node and imperfections in the lattice due to the fabrication process. The scaling laws of Gibson and Ashby predict a constant yield stress for all structures, yet this work demonstrates that tuning the microstructure of the constituent material and its dimensions at small length scales enables tuning the strength in the fabricated nanolattice structures [2]. Increasing the lattice angle from 45 deg to $60 \mathrm{deg}$, we observe an improvement over the lower angle case by $\sim 1.6$ times for multiple wall thicknesses and again show the tunable strength of the nanolattices as a result of material size effects.

The 45 deg nanolattices were nominally conformally coated with a $\sim 200 \mathrm{~nm}$-thick Au. TEM analysis revealed that these $200 \mathrm{~nm}$-thick films were more porous than samples with thicker walls. This is likely due to the porosity of the polymer onto which the $\mathrm{Au}$ is deposited; the $200 \mathrm{~nm}$-thick walls were the thinnest conformal layer possible with the sputtering technique and the pores within the thin walls are shown in Fig. 9. We discovered that thicker films had no observable pores, as revealed by TEM and ion channeling of $\sim 2 \mu \mathrm{m} \mathrm{n}$-Au thin films deposited under identical conditions. This decrease in porosity as $t$ increases is consistent with the mechanisms for polycrystalline film growth on a substrate where the nucleation sites grow to coalesce into a fully dense thin film [55]. The greater porosity impacts the mechanical response of the film because at low wall thicknesses the tubes of the nanolattices act as a thin film with defects rather than a fully dense constituent material. The films that comprise the nanolattice tubes are oriented at an angle to the loading direction, as defined by the lattice angle, so it is expected for the structures to be weaker when loaded in this configuration compared to loading applied along the length of the columnar grains. We propose this to be the cause of the large change in both yield stress and modulus as $t$ increases from $200 \mathrm{~nm}$ to $327 \mathrm{~nm}$. Ion channeling and TEM confirm that as the wall thickness increases, more grains span the thickness of the film, as shown in Fig. 10, however, there are single grains that may span the thickness of the nanolattice wall. Additionally the average height of the grains is on the order of $\sim 100 \mathrm{~nm}$ so it is suspected that for $t=200 \mathrm{~nm}$ there are fewer grains that span the thickness of the film; however, as $t$ increases more grains span the thickness and the behavior approaches that of the bulk constituent Au. The data for the 45 deg nanolattices show a significantly larger increase in yield strength as $t$ increased from 200 to $327 \mathrm{~nm}$ compared to the increase in yield strength as $t$ increased from 327 to $635 \mathrm{~nm}$ where there is no porosity. This suggests that the strength of the constituent material is approaching the bulk value for $t>327 \mathrm{~nm}$, which is also consistent with the literature for nanocrystalline nanopillars with increasing ratios of $(t / d)$ [24-26]. Existing models for thin films or nanopillars geometries are not directly applicable to this work since the stress state of the hollow nanolattice structures is complex and the microstructure varies as a function of wall thickness. Characterizing the deformation mechanism of the constituent material at varying wall thicknesses is the subject of future work to further understand the deformation mechanism of hollow metallic nanolattices at all length scales.

\section{Conclusions}

We fabricated hollow Au octahedral nanolattices with variable wall thicknesses and lattice angles and discovered that upon uniaxial compression, the structural and material size effects could be utilized to tune strength and stiffness. Without inducing a change in deformation mechanism of the lattice tubes, changes in the lattice angle can increase both the yield strength and modulus of the lattice by approximately an order of magnitude. Tuning the wall thickness of the nanolattices makes it possible to exploit material size effects of the small-scale constituent material, thus increasing the yield strength by a factor of 2. Classical mechanics of cellular solids predicts a constant strength and stiffness for all nanolattice geometries explored in this study, however, by utilizing material size effects, the strength of a lattice with a constant deformation mechanism and relative density can be increased by up to a factor of 2. Exploiting structural effects can provide up to an order of magnitude increase in strength and stiffness for a nanolattice with a constant deformation mechanism and relative density. We show that by exploiting the structural and material size effect parameters of nanolattices, the strength and stiffness of these cellular solids are no longer constant for a given deformation mechanism and relative density, as predicted by classical theories. By combining an understanding of structural mechanics and the behavior of small-scale materials, technology development is no longer limited by the constraints of existing cellular solids and new materials can be designed to meet the needs of emerging technology by exploiting structural and material size effects to tune mechanical properties.

\section{Acknowledgment}

The authors gratefully acknowledge the financial support from the National Science Foundation through NSF Graduate Research Fellowship of L.C.M. and Grant Nos. of J.R.G. (CMMI-1234364 and DMR-1204864). The authors would like to acknowledge Professor Lorenzo Valdevit at the University of California Irvine for the analytic model used to determine the deformation mechanism of the fabricated samples. The authors also acknowledge the critical support and infrastructure provided by the Kavli Nanoscience 
Institute at Caltech. The authors would also like to thank Z. Aitken and D. Jang for their help in TEM sample preparation/ analysis and L. Meza for SolidWorks images/discussion.

\section{References}

[1] Fleck, N. A., Deshpande, V. S., and Ashby, M. F., 2010, "Micro-Architectured Materials: Past, Present and Future," Proc. R. Soc. A, 466(2121), pp. $2495-2516$.

[2] Gibson, L. J., and Ashby, M. F., 1999, Cellular Solids: Structure and Properties, Cambridge University Press, Cambridge, UK.

[3] Wallach, J. C., and Gibson, L. J., 2001, "Mechanical Behavior of a ThreeDimensional Truss Material,” Int. J. Solids Struct., 38(40-41), pp. 7181-7196.

[4] Deshpande, V., and Fleck, N., 2001, "Collapse of Truss Core Sandwich Beams in 3-Point Bending," Int. J. Solids Struct., 38(36-37), pp. 6275-6305.

[5] Deshpande, V. S., Fleck, N. A., and Ashby, M. F., 2001, "Effective Properties of the Octet-Truss Lattice Material," J. Mech. Phys. Solids, 49(8), pp. 1747-1769.

[6] Zheng, X., Lee, H., Weisgraber, T. H., Shusteff, M., DeOtte, J., Duoss, E. B. Kuntz, J. D., Biener, M. M., Ge, Q., Jackson, J. A., Kucheyev, S. O., Fang, N. X., and Spadaccini, C. M., 2014, "Ultralight, Ultrastiff Mechanical Metamaterials," Science, 344(6190), pp. 1373-1377.

[7] Meza, L. R., Das, S., and Greer, J. R., 2014, "Strong, Lightweight, and Recoverable Three-Dimensional Ceramic Nanolattices," Science, 345(6202), pp. 1322-1326.

[8] Jang, D., Meza, L., Greer, F., and Greer, J., 2013, "Fabrication and Deformation of Three-Dimensional Hollow Ceramic Nanostructures," Nat. Mater., 12, pp. 893-898.

[9] Valdevit, L., Godfrey, S. W., Schaedler, T. A., Jacobsen, A. J., and Carter, W. B., 2013, "Compressive Strength of Hollow Microlattices: Experimental Characterization, Modeling, and Optimal Design," J. Mater. Res., 28(17), pp. $2461-2473$

[10] Torrents, A., Schaedler, T. A., Jacobsen, A. J., Carter, W. B., and Valdevit, L., 2012, "Characterization of Nickel-Based Microlattice Materials With Structura Hierarchy From the Nanometer to the Millimeter Scale," Acta Mater., 60(8), pp. 3511-3523.

[11] Schaedler, T. A., Jacobsen, A. J., Torrents, A., Sorensen, A. E., Lian, J., Greer, J. R., Valdevit, L., and Carter, W. B., 2011, "Ultralight Metallic Microlattices," Science, 334(6058), pp. 962-965.

[12] Ng, K. Y., Lin, Y., and Ngan, A. H. W., 2009, "Deformation of Anodic Aluminum Oxide Nano-Honeycombs During Nanoindentation," Acta Mater., 57(9), pp. 2710-2720.

[13] Wang, J., Evans, A. G., Dharmasena, K., and Wadley, H. N. G., 2003, "On the Performance of Truss Panels With Kagomé Cores," Int. J. Solids Struct., 40(25), pp. 6981-6988.

[14] Wadley, H. N. G., Fleck, N., and Evans, A. G., 2003, "Fabrication and Structural Performance of Periodic Cellular Metal Sandwich Structures," Compos. Sci. Technol., 63(16), pp. 2331-2343.

[15] Chiras, S., Mumm, D. R., Evans, A. G., Wicks, N., Hutchinson, J. W., Dharmasena, K., Wadley, H. N. G., and Fichter, S., 2002, "The Structural Performance of NearOptimized Truss Core Panels," Int. J. Solids Struct., 39(15), pp. 4093-4115.

[16] Hodge, A. M., Biener, J., Hayes, J. R., Bythrow, P. M., Volkert, C. A., and Hamza, A. V., 2007, "Scaling Equation for Yield Strength of Nanoporous Open-Cell Foams," Acta Mater., 55(4), pp. 1343-1349.

[17] Hutchinson, R. G., and Fleck, N. A., 2006, "The Structural Performance of the Periodic Truss," J. Mech. Phys. Solids, 54(4), pp. 756-782.

[18] Deshpande, V. S., Ashby, M. F., and Fleck, N. A., 2001, "Foam Topology: Bending Versus Stretching Dominated Architectures," Acta Mater., 49(6), pp. 1035-1040.

[19] Jennings, A. T., Burek, M. J., and Greer, J. R., 2010, "Microstructure Versus Size: Mechanical Properties of Electroplated Single Crystalline $\mathrm{Cu}$ Nanopillars," Phys. Rev. Lett., 104(13), p. 135503.

[20] Greer, J. R., and De Hosson, J. T. M., 2011, "Plasticity in Small-Sized Metallic Systems: Intrinsic Versus Extrinsic Size Effect,” Prog. Mater. Sci., 56(6), pp. 654-724.

[21] Greer, J. R., and Nix, W. D., 2005, "Size Dependence of Mechanical Properties of Gold at the Sub-Micron Scale," Appl. Phys. A, 80(8), pp. 1625-1629.

[22] Greer, J. R., Oliver, W. C., and Nix, W. D., 2005, "Size Dependence of Mechanical Properties of Gold at the Micron Scale in the Absence of Strain Gradients," Acta Mater., 53(6), pp. 1821-1830.

[23] Dou, R., and Derby, B., 2009, "A Universal Scaling Law for the Strength of Metal Micropillars and Nanowires," Scr. Mater., 61(5), pp. 524-527.

[24] Greer, J. R., Jang, D., and Gu, X. W., 2012, "Exploring Deformation Mechanisms in Nanostructured Materials," JOM, 64(10), pp. 1241-1252.

[25] Jang, D., and Greer, J. R., 2011, "Size-Induced Weakening and Grain Boundary-Assisted Deformation in $60 \mathrm{~nm}$ Grained Ni Nanopillars," Scr. Mater. 64(1), pp. 77-80.

[26] Gu, X. W., Loynachan, C. N., Wu, Z., Zhang, Y.-W., Srolovitz, D. J., and Greer, J. R., 2012, "Size-Dependent Deformation of Nanocrystalline Pt Nanopillars," Nano Lett., 12(12), pp. 6385-6392.

[27] Yang, B., Motz, C., Rester, M., and Dehm, G., 2012, "Yield Stress Influenced by the Ratio of Wire Diameter to Grain Size-A Competition Between the
Effects of Specimen Microstructure and Dimension in Micro-Sized Polycrystalline Copper Wires," Philos. Mag., 92(25-27), pp. 3243-3256.

[28] Rys, J., Valdevit, L., Schaedler, T. A., Jacobsen, A. J., Carter, W. B., and Greer, J. R., 2014, "Fabrication and Deformation of Metallic Glass Micro-Lattices," Adv. Eng. Mater., 16(7), pp. 889-896.

[29] Chen, D. Z., Jang, D., Guan, K. M., An, Q., Goddard, W. A., and Greer, J. R., 2013, "Nanometallic Glasses: Size Reduction Brings Ductility, Surface State Drives Its Extent," Nano Lett., 13(9), pp. 4462-4468.

[30] Chen, C. Q., Pei, Y. T., and De Hosson, J. T. M., 2010, "Effects of Size on the Mechanical Response of Metallic Glasses Investigated Through In Situ TEM Bending and Compression Experiments," Acta Mater., 58(1), pp. 189-200.

[31] Volkert, C. A., Donohue, A., and Spaepen, F., 2008, "Effect of Sample Size on Deformation in Amorphous Metals," J. Appl. Phys., 103(8), p. 083539.

[32] Valdevit, L., Jacobsen, A. J., Greer, J. R., and Carter, W. B., 2011, "Protocols for the Optimal Design of Multi-Functional Cellular Structures: From Hypersonics to Micro-Architected Materials," J. Am. Ceram. Soc., 94(S1), pp. s15-s34.

[33] Lian, J., Jang, D., Valdevit, L., Schaedler, T. A., Jacobsen, A. J., Carter, W. B., and Greer, J. R., 2011, "Catastrophic Versus Gradual Collapse of Thin-Walled Nanocrystalline Ni," Nano Lett., 11(10), pp. 4118-4125.

[34] Maloney, K. J., Roper, C. S., Jacobsen, A. J., Carter, W. B., Valdevit, L., and Schaedler, T. A., 2013, "Microlattices as Architected Thin Films: Analysis of Mechanical Properties and High Strain Elastic Recovery," APL Mater., 1(2), p. 022106.

[35] Jacobsen, A. J., Barvosa-Carter, W., and Nutt, S., 2007, "Micro-Scale Truss Structures Formed From Self-Propagating Photopolymer Waveguides," Adv. Mater., 19(22), pp. 3892-3896.

[36] Jacobsen, A. J., Barvosa-Carter, W., and Nutt, S., 2008, "Micro-Scale Truss Structures With Three-Fold and Six-Fold Symmetry Formed From SelfPropagating Polymer Waveguides," Acta Mater., 56(11), pp. 2540-2548.

[37] Meza, L. R., and Greer, J. R., 2014, "Mechanical Characterization of Hollow Ceramic Nanolattices," J. Mater. Sci., 49(6), pp. 2496-2508.

[38] Dietiker, M., Buzzi, S., Pigozzi, G., Löffler, J. F., and Spolenak, R., 2011, "Deformation Behavior of Gold Nano-Pillars Prepared by Nanoimprinting and Focused Ion-Beam Milling," Acta Mater., 59(5), pp. 2180-2192.

[39] Volkert, C. A., and Lilleodden, E. T., 2006, "Size Effects in the Deformation of Sub-Micron Au Columns," Philos. Mag., 86(33-35), pp. 5567-5579.

[40] Oh, S. H., Legros, M., Kiener, D., and Dehm, G., 2009, "In Situ Observation of Dislocation Nucleation and Escape in a Submicrometre Aluminium Single Crystal,” Nat. Mater., 8(2), pp. 95-100.

[41] Gu, X. W., and Greer, J. R., 2015, "Ultra-Strong Architected Cu MesoLattices," Extreme Mech. Lett., 2, pp. 7-14.

[42] Fischer, J., and Wegener, M., 2013, "Three-Dimensional Optical Laser Lithography Beyond the Diffraction Limit," Laser Photonics Rev., 7(1), pp. 22-44.

[43] Sun, H., and Kawata, S., 2004, "Two-Photon Photopolymerization and 3D Lithographic Microfabrication," NMR, 3D Analysis, Photopolymerization (Advances in Polymer Science, Vol. 170), Springer-Verlag, Berlin, pp. 169-274.

[44] Xiong, W., Zhou, Y. S., He, X. N., Gao, Y., Mahjouri-Samani, M., Jiang, L., Baldacchini, T., and Lu, Y. F., 2012, "Simultaneous Additive and Subtractive Three-Dimensional Nanofabrication Using Integrated Two-Photon Polymerization and Multiphoton Ablation," Light: Sci. Appl., 1(4), p. e6.

[45] LaFratta, C. N., Fourkas, J. T., Baldacchini, T., and Farrer, R. A., 2007, "Multiphoton Fabrication," Angew. Chem., Int. Ed. Engl., 46(33), pp $6238-6258$

[46] Jeon, S., Malyarchuk, V., Rogers, J. A., and Wiederrecht, G. P., 2006, "Fabricating Three-Dimensional Nanostructures Using Two Photon Lithography in a Single Exposure Step," Opt. Express, 14(6), pp. 2300-2308.

[47] Sun, H.-B., Matsuo, S., and Misawa, H., 1999, "Three-Dimensional Photonic Crystal Structures Achieved With Two-Photon-Absorption Photopolymerization of Resin,” Appl. Phys. Lett., 74(6), pp. 786-788.

[48] Tétreault, N., von Freymann, G., Deubel, M., Hermatschweiler, M., PérezWillard, F., John, S., Wegener, M., and Ozin, G. A., 2006, "New Route to Three-Dimensional Photonic Bandgap Materials: Silicon Double Inversion of Polymer Templates," Adv. Mater., 18(4), pp. 457-460.

[49] Montemayor, L. C., Meza, L. R., and Greer, J. R., 2014, "Design and Fabrication of Hollow Rigid Nanolattices Via Two-Photon Lithography," Adv. Eng. Mater., 16(2), pp. 184-189.

[50] Thornton, J. A., 1975, "Influence of Substrate Temperature and Deposition Rate on Structure of Thick Sputtered Cu Coatings," J. Vac. Sci. Technol., 12(4), pp. 830-835.

[51] Thornton, J. A., 1986, "The Microstructure of Sputter-Deposited Coatings," J. Vac. Sci. Technol., A, 4(6), pp. 3059-3065.

[52] Thornton, J. A., 1974, "Influence of Apparatus Geometry and Deposition Conditions on the Structure and Topography of Thick Sputtered Coatings," J. Vac. Sci. Technol., 11(4), pp. 666-670.

[53] Valdevit, L., Dec. 2013, private communication.

[54] Oliver, W., and Pharr, G., 1992, "An Improved Technique for Determining Hardness and Elastic Modulus Using Load and Displacement Sensing Indentation Experiments," J. Mater. Res., 7(6), pp. 1564-1583.

[55] Thompson, C. V., 2000, "Structure Evolution During Processing of Polycrystalline Films," Annu. Rev. Mater. Sci., 30, pp. 159-190. 